\title{
Identifizierung toxischer Verbindungen in Sedimenten: Ansätze zur Integration von Wirkung und Bioverfügbarkeit
}

\author{
Werner Brack • Nicole Bandow $\cdot$ Katrin Schwab $\cdot$ Tobias Schulze $\cdot$ Georg Streck
}

Eingegangen: 11. März 2009/Akzeptiert: 29. April 2009/Online veröffentlicht: 19. Mai 2009

(C) Springer-Verlag 2009

\begin{abstract}
Zusammenfassung Sedimente spielen eine wichtige Rolle für die Funktionen aquatischer Ökosysteme, fungieren aber auch als Senke, Speicher und Quelle für lipophile Schadstoffe und Metalle. Wirkungsorientierte Analytik (EDA) ist ein geeignetes Werkzeug, um besonders problematische Stoffe zu identifizieren. Um Missinterpretationen und falsche Prioritätensetzung zu vermeiden, sollte dabei neben den eigentlichen Effekten die Bioverfügbarkeit berücksichtigt werden. Bioverfügbarkeit wird dabei als komplexer Prozess betrachtet, der letztendlich dazu führt, dass ein bisher partikelgebundenes Molekül am Wirkort im Organismus ankommt und dort Schäden verursachen kann. Um diesen Prozess in der EDA operativ berücksichtigen zu können, kann er in mehrere Teilprozesse zerlegt werden. Dies beinhaltet die Desorption vom Sediment und damit die biologische Zugänglichkeit, die Gleichgewichtsverteilung desorbierbarer Stoffe zwischen Sediment, Porenwasser und Organismus entsprechend der Aktivität in den verschiedenen Phasen und die toxikologische Bioverfügbarkeit als Resultat toxikokinetischer Prozesse wie Resorption, Transport, Metabolisierung und Ausscheidung. Die biologische Zugänglichkeit als Resultat der Desorptionskinetik lässt sich durch den Einsatz milder Extraktionsverfahren z. B. mit TENAX in die EDA einbeziehen. Die Gleichgewichtsverteilung im System Sediment-Wasser-Organismus kann durch verteilungsbasierte Dosierung simuliert werden. Erste Ergebnisse mit diesen Verfahren zeigen, dass bei Einbeziehung von Bioverfügbar-
\end{abstract}

Verantwortliche Herausgeber: Jan Schwarzbauer · Peter Heininger · Evelyn Claus

W. Brack $(\square) \cdot$ N. Bandow $\cdot$ K. Schwab $\cdot$ T. Schulze $\cdot$ G. Streck

Helmholtz Zentrum für Umweltforschung - UFZ,

Department für Wirkungsorientierte Analytik,

Permoserstraße 15, 04318 Leipzig, Deutschland

E-Mail: werner.brack@ufz.de keit polare Sedimentkontaminanten relativ zu klassischen unpolaren Problemstoffen wie Polyzyklischen Aromatischen Kohlenwasserstoffen deutlich an Bedeutung gewinnen.

Schlüsselwörter Wirkungsorientierte Analytik · Partikelgebundene Schadstoffe · Biologische Zugänglichkeit $\cdot$ Verteilungsbasierte Dosierung $\cdot$ Aktivität

\section{Identification of toxicants in sediments:} Approaches for integration of effects and bioavailability

Abstract Sediments play an important role for aquatic ecosystem functions. However, they also act as sink, storage and source of lipophilic toxicants and metals. Effect-directed analysis (EDA) is a powerful tool to identify compounds causing adverse effects. In order to avoid misinterpretations and biased prioritization bioavailability needs to be considered together with effects. Bioavailability is a complex process finally resulting in the transfer of a so far particle bound molecule to the target location within the organism where it causes an effect. In order to operationalize this concept for EDA it can be divided into several partial processes. These include desorption from sediments and thus bioaccessibility, equilibrium partitioning of desorbable compounds between sediment, water and organisms driven by activity, and toxicological bioavailability as a result of toxicokinetics including resorption, transport, metabolization and excretion. Bioaccessibility is based on desorption kinetics and can be simulated with mild extraction methods e.g. using TENAX. Equilibrium partitioning can be simulated with partition-based dosing techniques. First results with these approaches indicate that consideration of bioavailability increases the significance of polar sedimentassociated toxicants relative to classical non-polar contaminants such as polycyclic aromatic hydrocarbons. 
Keywords Effect-directed analysis · Particle-bound toxicants $\cdot$ Bioaccessibility $\cdot$ Partition-based dosing . Activity

\section{Wirkungsorientierte Analytik in Sedimenten}

Sedimente spielen eine wichtige Rolle für aquatische Ökosysteme und deren Leistungen für die menschliche Gesellschaft. Als bedeutendste Senke im Gewässer akkumulieren Sedimente lipophile organische Stoffe und Metalle und können über die Nahrungsnetze oder physikalische Remobilisierung selbst bei einer Verbesserung der Wasserqualität noch lange als Schadstoffquelle für benthische Organismen, aber auch für das gesamte Ökosystem, fungieren. Für das Gefährdungspotenzial spielen dabei die bioverfügbare Menge und die Wirkung der Stoffe die entscheidende Rolle.

Da Sedimente in der Regel mit einer Vielzahl von Stoffen unterschiedlicher Wirkung und Bioverfügbarkeit belastet sind, können auftretende Wirkungen mithilfe von TargetAnalytik ausgewählter Stoffe oft nicht erklärt werden. Hier hilft die wirkungsorientierte Analytik (EDA) als Kombination von biologischer Wirkungstestung, Fraktionierung und chemischer Identifizierung, das Augenmerk auf die Stoffe zu lenken, die tatsächlich für Schadeffekte verantwortlich sind (Brack 2003). Neue Fraktionierungs- und Identifizierungstechniken konnten hier die Diagnosefähigkeit der EDA deutlich verbessern (Lübcke-von Varel et al. 2008; Meinert et al. 2007; Schymanski et al. 2008). Neben der Toxizität bestimmt im Ökosystem die Bioverfügbarkeit, welche Stoffe ein Risiko für die Organismen darstellen. Dabei wird durch Nichtberücksichtigung der Bioverfügbarkeit nicht nur das Gefährdungspotenzial überschätzt - was für die Priorisie- rung von Stoffen und Fraktionen in der EDA von untergeordneter Bedeutung ist - sondern auch der relative Beitrag unterschiedlich bioverfügbarer Verbindungen falsch eingeschätzt. Daher wurden in jüngster Zeit verstärkt Verfahren zur Integration bioverfügbarkeitsorientierter Ansätze in die EDA entwickelt (Schwab et al. 2009; Bandow et al. 2009). Eine ausführliche Darstellung der relevanten Konzepte wurde kürzlich veröffentlicht (Brack et al. 2009).

\section{Konzepte zur Einbeziehung der Bioverfügbarkeit}

„Bioverfügbarkeit ist der Grad, in dem Inhaltsstoffe von Böden und Sedimenten durch Organismen aufgenommen oder metabolisiert werden können oder für Wechselwirkungen mit dem biologischen System verfügbar sind“ (übersetzt aus ISO 11074). Bioverfügbarkeit ist offensichtlich ein komplexes Phänomen, das als Ganzes nur schwer in Handlungsanweisungen für die EDA übertragen werden kann. Stattdessen ist es sinnvoll, diesen Prozess in verschiedene Teilschritte zu zerlegen (Abb. 1) (Harmsen 2007). Ein erster Teilprozess ist die Desorption von der Sedimentmatrix. Nur Moleküle, die in relevanten Zeiträumen von dieser desorbieren können, stehen für eine Akkumulation in biologischen Systemen zur Verfügung. Dieser schnell desorbierbare Anteil lässt sich typischerweise in wässriger Suspension mittels konkurrierender starker Sorbentien wie TENAX extrahieren (Cornelissen et al. 1998) und kann als Ausgangspunkt einer EDA dienen (Schwab und Brack 2007). Bei den extrahierten Anteilen spricht man von biologisch zugänglichen Anteilen. In der englischsprachigen Literatur wurde hierfür der Begriff „,bioaccessibility“ geprägt. Derartige Extraktionsverfahren simulieren nicht die Aufnahme in Organismen, können aber
Abb. 1 Allgemeines Schema zur Bioverfügbarkeit, verändert nach Harmsen (2007)

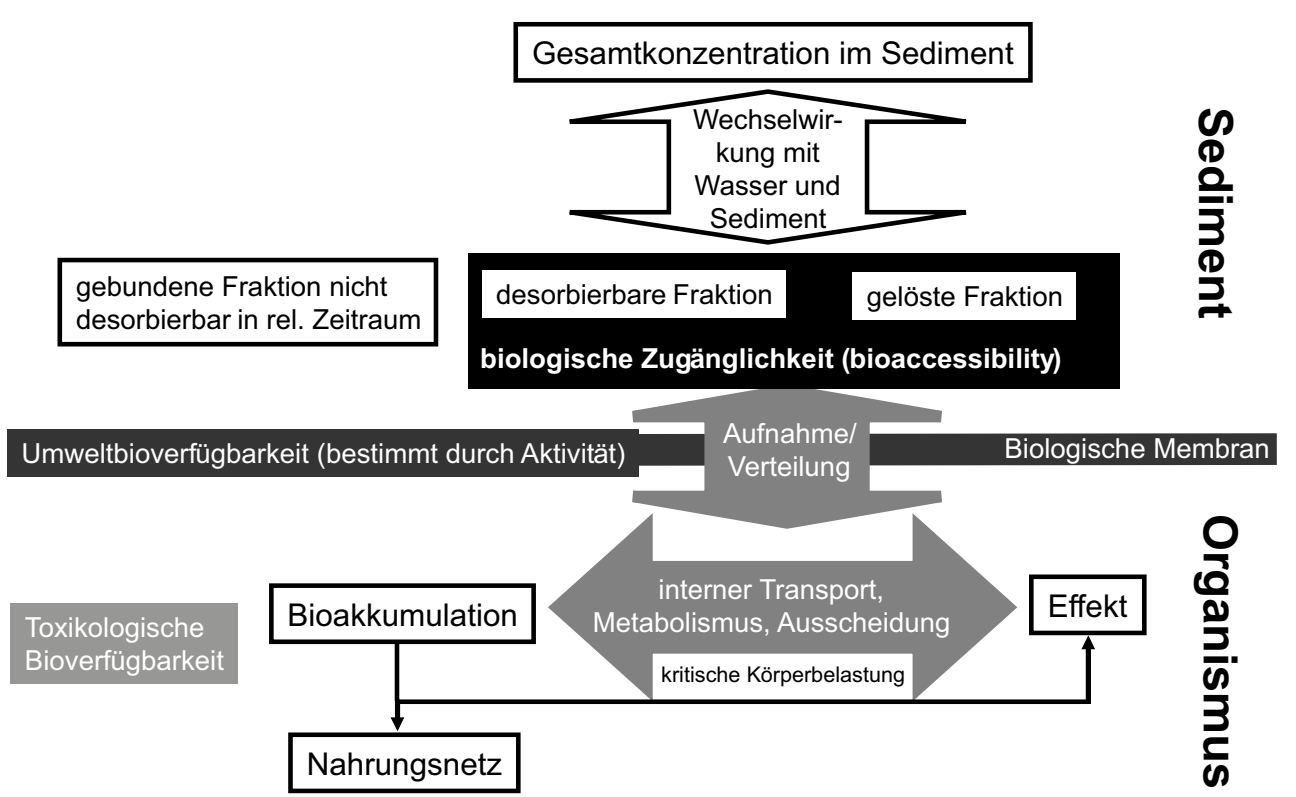


sehr fest gebundene Stoffanteile, wie beispielsweise rußgebundene Polyzyklische Aromatische Kohlenwasserstoffe (PAHs), die oft nur in Zeiträumen von Jahren bis Jahrtausenden desorbieren (Jonker et al. 2005) und deshalb für die Gefährdung benthischer Organismen ohne Bedeutung sind, von der EDA ausschließen. Eine Reihe von Untersuchungen hat gezeigt, dass für eine Akkumulation in benthischen Organismen nur die Stoffanteile zur Verfügung stehen, die ausreichend schnell von Sedimentpartikeln desorbieren.

Ein zweiter Teilprozess ist die Aufnahme von desorbierbaren und gelösten Molekülen in den Organismus durch Gleichgewichtsverteilung im System Sediment-Wasser-Biota gemäß der chemischen Aktivität in den verschiedenen Kompartimenten (Reichenberg und Mayer 2006). Dieser Prozess, der auch als Umweltbioverfügbarkeit bezeichnet werden kann (Harmsen 2007), kann mithilfe verteilungsbasierter Dosierungstechniken simuliert werden (Brown et al. 2001; Bandow et al. 2009). Hierbei werden organische Extrakte, Fraktionen oder Einzelverbindungen auf ein festes Sorbens aufgetragen und in das Testsystem eingebracht. Die Konzentration im Medium ergibt sich aus der stoffspezifischen Gleichgewichtsverteilung zwischen Sorbens und Medium. Entscheidende Vorteile gegenüber herkömmlichen Dosierungstechniken, z. B. mittels DMSO, sind die Konzentrationseinstellung entsprechend der Verteilungskoeffizienten analog der Verteilung zwischen der organischen Substanz im Sediment und dem Porenwasser sowie die Einstellung einer konstanten Konzentration über die gesamte Testdauer durch die Kompensation von Verlusten durch Sorption, Abbau oder Verdunstung. Ein neues, einfaches und kosteneffizientes Dosierungssystem für die EDA mit Grünalgen wurde hierfür entwickelt (Bandow et al. 2009).

An der biologischen Zugänglichkeit orientierte Extraktionsverfahren und verteilungsbasierte Dosierung sind beide mit dem Ansatz der Wirkungsorientierten Analytik verknüpfbar und ergänzen sich, da sie unterschiedliche Teilprozesse des Phänomens Bioverfügbarkeit beschreiben (Brack et al. 2009).

Der dritte Teilprozess, die toxikologische Bioverfügbarkeit, fokussiert auf Prozesse im Organismus wie Transport, Metabolisierung und Ausscheidung, die letztendlich die Konzentration am Wirkort bestimmen. Diese in den exponierten Organismen ablaufenden Vorgänge können nur durch EDA in biologischem Gewebe selbst berücksichtigt werden. Auch wenn es hier schon sehr erfolgreiche Arbeiten wie die Identifizierung von östrogenen Verbindungen in Fischgalle gegeben hat (Houtman et al. 2004), bleibt die Anwendbarkeit doch begrenzt. Neben praktischen Nachteilen, wie sehr kleine zur Verfügung stehende Probenmengen, gibt es eine Reihe von konzeptionellen Nachteilen. Die Akkumulation von Stoffen ist abhängig von der Toxikokinetik im entsprechenden Organismus und damit von der Spezies und dem Lebensstadium. Eine Übertragung auf andere Organismen ist nur begrenzt möglich. Viele Stoffe werden im Organismus metabolisiert und können im Gewebe nicht wiedergefunden werden, obwohl sie Schadwirkungen auslösen.

\section{Bioverfügbarkeit und Priorisierung wirksamer Fraktionen}

Bei der EDA von Sedimenten spielen für viele toxikologische Endpunkte PAHs eine zentrale Rolle. Vertreter dieser Stoffgruppe zeigen aufgrund ihrer Lipophilie hohe Basistoxizität, sind phototoxisch, mutagen und induzieren Arylhydrocarbon-Rezeptor-vermittelte Wirkung. Sie sind ubiquitär vorhanden und akkumulieren in Böden und Sedimenten. Allerdings sind PAH aufgrund ihrer Lipophilie und ihrer sehr starken Bindung, z.B. an Rußpartikel, und
Abb. 2 Hemmung der Zellvermehrung von Scenedesmus vacuolatus durch Fraktionen aus Sedimenttotalextrakten (ASE) und an der biologischen Zugänglichkeit orientierten TENAX-Extrakten. Die Fraktionen koeluieren mit Polychlorierten Biphenylen (PCBs), Polychlorierten Dibenzo-p-dioxinen und furanen (PCDD/Fs), Polyzyklischen Aromatischen Kohlenwasserstoffen (PAHs) und polareren Komponenten (verändert nach Schwab et al. 2009)

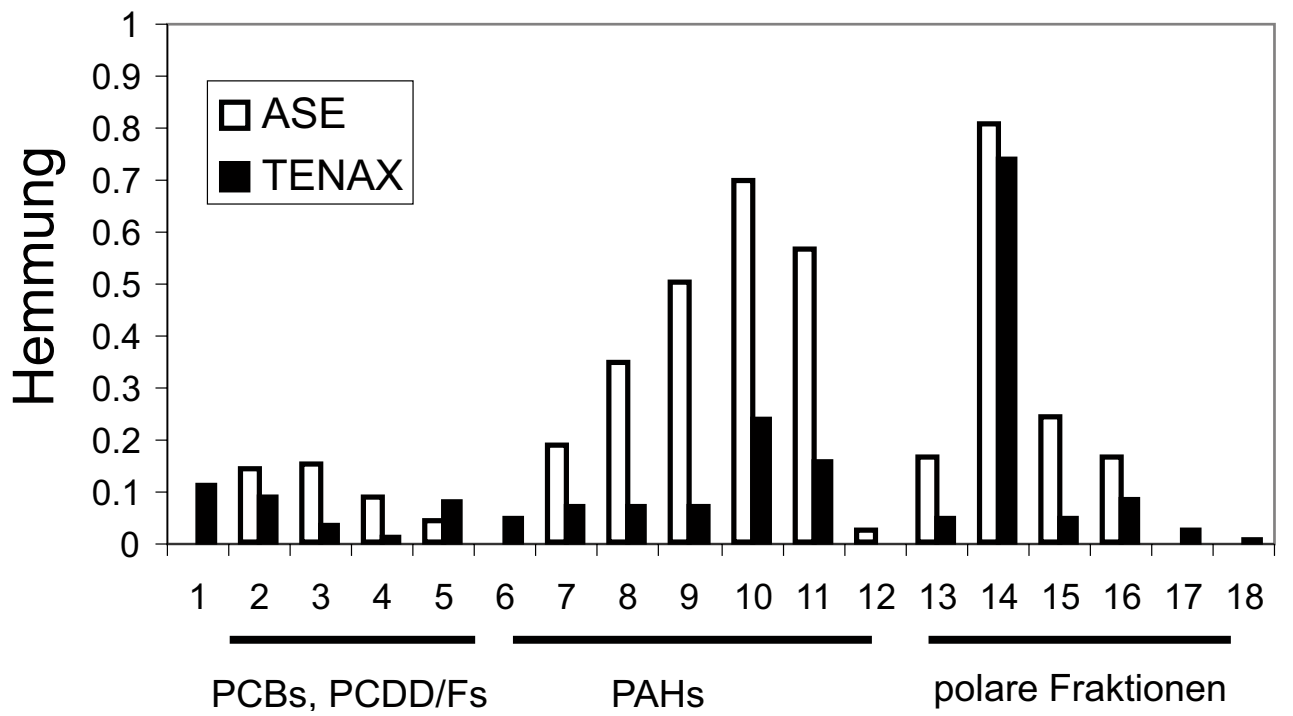


Abb. 3 Zellvermehrungshemmung von Scenedesmus vacuolatus (wei $\beta$ : 0-20\%, grau: $20-50 \%$, schwarz: $>50 \%$ ) exponiert gegenüber Fraktionen von drei verschiedenen Sedimentextrakten (S1-S3) mittels verteilungsbasierter Dosierung (PBD) und Dosierung mit Dimethylsulfoxid (DMSO)

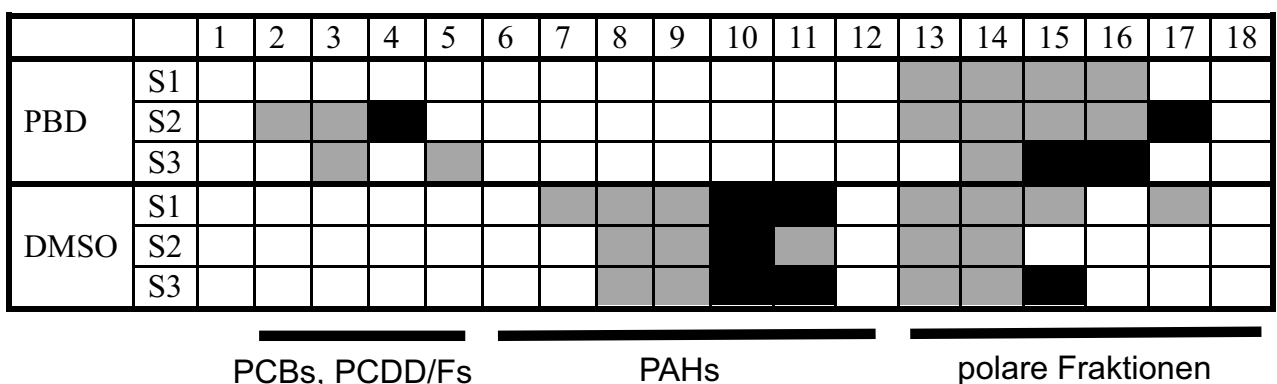

damit sehr langsamen Desorptionskinetik oft nur wenig bioverfügbar (Jonker et al. 2005). Wird dies bei der EDA nicht berücksichtigt, kann es bei der Priorisierung wirksamer Fraktionen im Hinblick auf das Gefährdungspotenzial zu Fehleinschätzungen kommen. Dies konnte in kürzlich veröffentlichten Arbeiten anhand verschiedener Sedimente gezeigt werden.

Dabei wurden Sedimente sowohl einer erschöpfenden beschleunigten Lösemittelextraktion (ASE) unterzogen als auch an der biologischen Zugänglichkeit orientiert mit TENAX extrahiert und nach Fraktionierung auf ihre Toxizität gegenüber der Grünalge Scenedesmus vacuolatus untersucht (Schwab et al. 2009). Während PAH-Fraktionen in den meisten der untersuchten Sedimente nach ASE die Wirkung dominieren, reduziert die Einbeziehung der biologischen Zugänglichkeit durch TENAX-Extraktion ihre Bedeutung deutlich. Polarere Fraktionen gewinnen dagegen an relativer Bedeutung (Abb. 2).

Ähnliche Ergebnisse wurden mit verteilungsbasierter Dosierung mittels Silikonrührstäbchen erzielt (Bandow et al. 2009). Während polare Fraktionen unabhängig von der Dosierungstechnik in allen untersuchten Sedimenten eine wichtige Rolle spielten, wurde die Toxizität der PAHFraktionen nur bei der Dosierung mit DMSO beobachtet (Abb. 3). Bei Simulation der Verteilungsprozesse im Sediment-Wasser-Biota-System durch Dosierung der PAHFraktionen mittels Silikonrührstäbchen wurde keine Hemmung der Zellvermehrung von Scenedesmus vacuolatus beobachtet.

\section{Schlussfolgerungen und Ausblick}

Die Berücksichtigung der Bioverfügbarkeit bei der Identifizierung gefährlicher Stoffe in Sedimenten hilft dabei, eine realistischere Priorisierung von Fraktionen und Substanzen zu erreichen. Während EDA in Gewebe nur im Einzelfall erfolgversprechend sein dürfte, lässt die Kombination von an der biologischen Zugänglichkeit orientierten Extraktion mit verteilungsbasierter Dosierung eine realistischere Prioritätensetzung wirksamer Fraktionen und Stoffe unter Berücksichtigung von Exposition und Wirkung erwarten. Obwohl bereits eine große Zahl von Arbeiten zur Extraktion z.B. mit TENAX publiziert wurde, beschränken sich die meisten dieser Arbeiten auf einen kleinen Satz unpolarer Verbindungen, mehrheitlich PAHs. Eine solide Methodenvalidierung für andere Substanzgruppen steht noch aus. Inwieweit die Sorptionsdomäne von TENAX tatsächlich das gesamte in Sedimenten relevante Schadstoffspektrum abdecken kann, oder ob hier andere Sorbentien zum Einsatz kommen müssen, ist Gegenstand der Forschung.

\section{Literatur}

Bandow N, Altenburger R, Lübcke-von Varel U, Paschke A, Streck G, Brack W (2009) Partition-based dosing: An approach to include bioavailability in the effect-directed analysis of contaminated sediment samples. Environ Sci Technol (im Druck)

Brack W (2003) Effect-directed analysis: a promising tool for the identification of organic toxicants in complex mixtures. Anal Bioanal Chem 377:397-407

Brack W, Bandow N, Schwab K, Schulze T, Streck G (2009) Bioavailability in effect-directed analysis of organic toxicants in sediments. TrAC Trends in Analytical Chemistry 28:543-549

Brown RS, Akhtar P, Akerman J, Hampel L, Kozin IS, Villerius LA, Klamer HJC (2001) Partition controlled delivery of hydrophobic substances in toxicity tests using poly(dimethylsiloxane) (PDMS) films. Environ Sci Technol 35:4097-4102

Cornelissen G, Rigterink H, Ferdinandy MMA, van Noort PCM (1998) Rapidly desorbing fractions of PAHs in contaminated sediments as a predictor of the extent of bioremediation. Environ Sci Technol 32:966-970

Harmsen J (2007) Measuring bioavailability: From a scientific approach to standard methods. J Environ Qual 36:1420-1428

Houtman CJ, Van Oostven AM, Brouwer A, Lamoree MH, Legler J (2004) Identification of estrogenic compounds in fish bile using bioassay-directed fractionation. Environ Sci Technol 38:6415-6423

ISO 11074 (2005) Soil quality-Vocabulary. ISO, Geneva, Switzerland.

Jonker MTO, Hawthorne SB, Koelmans AA (2005) Extremely slowly desorbing polycyclic aromatic hydrocarbons from soot and sootlike materials: Evidence by by supercritical fluid extraction. Environ Sci Technol 39:7889-7895

Lübcke-von Varel U, Streck G, Brack W (2008) Automated fractionation procedure for polycyclic aromatic compounds in sediment extracts on three coupled normal-phase high-performance liquid chromatography columns. J Chrom A 1185:31-42

Meinert C, Moeder M, Brack W (2007) Fractionation of technical p-nonylphenol with preparative capillary gas chromatography. Chemosphere 70:215-223 
Reichenberg F, Mayer P (2006) Two complementary sides of bioavailability: accessibility and chemical activity of organic contaminants in sediments and soils. Environ Toxicol Chem 25:1239-1245

Schwab K, Brack W (2007) Large volume TENAX ${ }^{\circledR}$ extraction of the bioaccessible fraction of sediment-associated organic compounds for a subsequent effect-directed analysis. J Soils Sediments $7: 178-186$
Schwab K, Altenburger R, Lübcke-von Varel U, Streck G, Brack W (2009) Effect-directed analysis of sediment-associated algal toxicants at selected hot spots in the river Elbe basin with a special focus on bioaccessibility. Environ Toxicol Chem (im Druck)

Schymanski EL, Meinert C, Meringer M, Brack W (2008) The use of MS classifiers and structure generation to assist in the identification of unknowns in effect-directed analysis. Analyt Chim Acta 615:136-147 